\title{
Primeiro censo brasileiro em cirurgia refrativa
}

\author{
First Brazilian refractive surgery survey
}

\author{
Gustavo Victor ${ }^{1}$ \\ Andreia Urbano ${ }^{2}$ \\ Sônia Marçal $^{3}$ \\ Ricardo Porto ${ }^{4}$ \\ Claudia Maria Francesconi ${ }^{5}$ \\ Adriana dos Santos Forseto ${ }^{6}$ \\ Breno Barth ${ }^{7}$ \\ Milton Ruiz Alves ${ }^{8}$ \\ Walton Nosé ${ }^{9}$
}

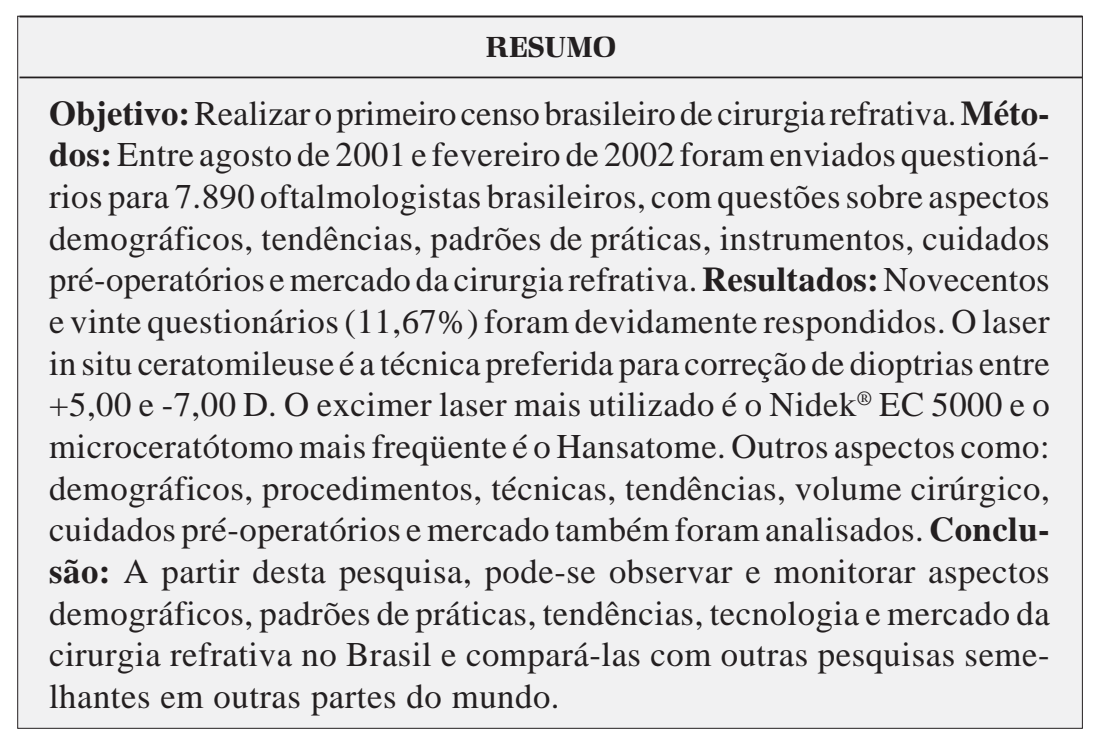

Descritores: Erros de refração/cirurgia; Cirurgia a laser; Ceratomileuse assistida po excimer laser in situ; Questionários; Oftalmologia/estatísticas \& dados numéricos; Condutas na prática dos médicos/estatísticas \& dados numéricos; Coleta de dados; Brasil

\section{INTRODUÇÃO}

Trabalho realizado no Eye Clinic Day Hospital.

${ }^{1}$ Pós-graduando, nível Doutorado pela Faculdade de Medicina de Ribeirão Preto da Universidade de São Paulo (USP). Ribeirão Preto (SP).

${ }_{2}^{2}$ Pós-graduando, nível Doutorado pela Universidade Federal de São Paulo (UNIFESP). São Paulo (SP).

${ }^{3}$ Oftalmologista da Eye Clinic Day Hospital. São Paulo (SP).

${ }^{4}$ Oftalmologista da Eye Clinic Day Hospital. São Paulo (SP).

${ }^{5}$ Doutora em Oftalmologia pela UNIFESP. São Paulo (SP).

${ }^{6}$ Doutora em Oftalmologia pela UNIFESP. São Paulo (SP).

Doutor em Oftalmologia pela Faculdade de Medicina da USP. São Paulo (SP).

${ }^{8}$ Livre Docente da USP. São Paulo (SP).

9 Titular do Departamento de Oftalmologia da Universidade Metropolitana de Santos (UNIMES). Santos (SP). Livre Docente da UNIFESP. São Paulo (SP).

Endereço para correspondência: Gustavo Victor Av. República do Líbano 1034 - São Paulo (SP) CEP 04502-001.

E-mail: gustavo.victor@eyeclinic.com.br

Recebido para publicação em 01.04.2005

Versão revisada recebida em 20.09.2005

Aprovação em 03.10.2005

Nota Editorial: Depois de concluída a análise do artigo sob sigilo editorial e com a anuência dos Drs. Belquiz Rodrigues do Amaral Nassaralla e Wallace Chamon sobre a divulgação de seu nome como revisor, agradecemos sua participação neste processo.

Os autores não têm nenhum interesse comercial no produto em estudo.

\section{MÉTODO}

Em agosto de 2001, uma carta e um questionário sobre informações dos oftalmologistas, prática padrão, técnicas cirúrgicas, equipamentos e instrumentação, taxas de complicações e cuidados per-operatórios foram enviados para 7.890 oftalmologistas brasileiros. As perguntas foram propostas pelos autores em associação com a SBCR. Na época, um dos autores era presidente da SBCR. Os oftalmologistas foram motivados a responder e enviar o questionário para a sede da SBCR. A logística de distribuição em todo território 
nacional e seus custos foi feitos pela Alcon ${ }^{\circledR}$ Brasil. A data limite para envio das respostas foi 26/02/2002. Todos os questionários respondidos e enviados a SBCR foram analisados. Com ajuda do software Microsoft Excel ${ }^{\circledR} 2000$, construiu-se um banco de dados e realizou-se a análise estatística.

\section{RESULTADOS}

Novecentos e vinte questionários $(11,67 \%)$ foram devidamente respondidos e enviados à sede da SBCR até a data limite de entrega.

\section{GERAL}

\section{Demográficos}

A maior parte dos respondentes tinha idade igual ou menor que 35 anos (22,6\%); 16,5\% tinham entre 45 e 49 anos; $16,2 \%$ entre 50 e 54 anos; $15,4 \%$ entre 40 e 44 anos; $5,2 \%$ entre 55 e 59 anos; $5,2 \%$ entre 60 e 64 anos; e, 2,2\% tinham idade igual ou maior que 64 anos. Entre os respondentes, $75 \%$ eram do sexo masculino. Vinte e um por cento dos respondentes tinham até 10 anos de formado; $18,9 \%$ tinham entre 20 e 25 anos de formado; $14,6 \%$ entre 15 e 20 anos; $14,6 \%$ entre 25 e 30 anos; $3,4 \%$ entre 30 e 35 anos; $2 \%$ entre 35 e 40 anos; e, $1,8 \%$ tinham mais de 40 anos de formado. Do total dos oftalmologistas, $7,2 \%$ já se submeteram a algum tipo de cirurgia refrativa. Vinte e nove por cento dos respondentes afirmaram ter entre 1 e 3 anos de experiência em cirurgia refrativa; $27,5 \%$ tinham entre 4 e 6 anos de experiência; $14,4 \%$ entre 7 e 10 anos; $11,5 \%$ tinham mais de 15 anos de experiência em CR; 10,8\% tinham entre 11 e 15 anos; e, 6,8\% dos respondentes afirmaram ter menos de 1 ano de experiência em CR.

\section{Número de cirurgia refrativa, tipos de cirurgia refrativa e local onde as realizam}

A maior parte dos respondentes (30\%) realiza menos de 5 CRs por mês, enquanto $28,4 \%$ realizam entre 5 a 10 CRs por mês; $16,8 \%$ realizam mais de 30 CRs por mês; $12,1 \%$ realizam entre 11 e 20 CRs por mês; e, 5,7\% realizam entre 21 e 30 CRs por mês. O quadro 1 mostra a distribuição dos tipos de CRs na prática diária dos entrevistados. A maioria $(53,7 \%)$ dos entrevistados não possui centro cirúrgico refrativo, enquanto $37,8 \%$ possuem sociedade em algum centro cirúrgico refrativo, e $8,5 \%$ possuem seu próprio centro cirúrgico refrativo. Quanto ao local onde realizam as cirurgias refrativas, 54,2\% dos respondentes realizam suas cirurgias refrativas em uma clínica oftalmológica, 25,5\% realiza-as em algum hospital, 7,7\% realizam em clínicas próprias, e 2,6\% realizam em ambos os lugares, clínica oftalmológica e hospital.

\section{LASIK}

\section{Indicação e exames pré-operatórios}

A idade média mínima para quem os entrevistados indicam a cirurgia refrativa foi de 20,42 $\pm 1,9$ anos. O quadro 2 mostra para qual dioptria (D) os entrevistados indicam LASIK, considerando os tipos de erros refracionais (miopia, hipermetropia, astigmatismo miópico e astigmatismo hipermetrópico). No exame pré-operatório, 99,3\% dos respondentes realizam topografia corneana computadorizada, 96,7\% realizam refração estática, 97,4\% realizam paquimetria ultra-sônica do centro da córnea, $56,5 \%$ realizam paquimetria ultra-sônica da periferia corneana, $95,0 \%$ realizam exame de mapeamento de retina, $13,7 \%$ avaliam a profundidade da câmara anterior, $20,9 \%$ aferem o comprimento axial do globo ocular, $24,5 \%$ realizam microscopia especular da córnea, 97,3\% aferem a pressão ocular (PIO), 63,1\% aferem subjetivamente o diâmetro pupilar, 4,1 realizam pupilometria infravermelha, $14,1 \%$ realizam teste de Schirmer e $4,1 \%$ realizam outros tipos de exames no pré-operatório.

\section{Tecnologia}

O excimer laser mais utilizado é o Nidek ${ }^{\circledR}$ EC-5000 (42,6\%), seguido pelos modelos Bausch \& Lomb 217 Technolas ${ }^{\circledR}$ e Visx S2/S3 ${ }^{\circledR}$ ambos com $13,1 \%$, em seguida estão os modelos Alcon

\begin{tabular}{|c|c|c|c|c|c|c|c|c|c|}
\hline \multicolumn{10}{|c|}{ Quadro 1. Distribuição dos tipos de cirurgia refrativa na prática diária dos entrevistados } \\
\hline $1-10 \%$ & $9,5 \%$ & $7,8 \%$ & $33,5 \%$ & $6,3 \%$ & $5,2 \%$ & $17,7 \%$ & $6,1 \%$ & $32,0 \%$ & $9,0 \%$ \\
\hline $11-20 \%$ & $0,2 \%$ & $0,6 \%$ & $2,2 \%$ & $0,8 \%$ & $2,8 \%$ & $2,0 \%$ & $0,4 \%$ & $11,3 \%$ & $0,8 \%$ \\
\hline $21-30 \%$ & - & - & $0,9 \%$ & $0,2 \%$ & $2,4 \%$ & $0,9 \%$ & - & $5,0 \%$ & $0,6 \%$ \\
\hline $51-60 \%$ & $0,2 \%$ & - & $0,4 \%$ & - & $3,0 \%$ & - & $0,2 \%$ & $2,0 \%$ & - \\
\hline $61-70 \%$ & - & - & $0,4 \%$ & $0,2 \%$ & $3,0 \%$ & $0,2 \%$ & $0,2 \%$ & $0,7 \%$ & $0,2 \%$ \\
\hline $71-80 \%$ & - & - & - & - & $8,7 \%$ & $0,4 \%$ & - & $2,5 \%$ & $0,2 \%$ \\
\hline $81-90 \%$ & - & - & - & - & $20,6 \%$ & $0,6 \%$ & $0,2 \%$ & $2,3 \%$ & - \\
\hline $91-100 \%$ & - & - & $0,6 \%$ & $0,4 \%$ & $38,7 \%$ & $1,1 \%$ & - & $4,6 \%$ & $0,8 \%$ \\
\hline
\end{tabular}




\begin{tabular}{|c|c|c|c|c|}
\hline \multicolumn{5}{|c|}{ Quadro 2. Para qual dioptria os entrevistados indicam LASIK, considerando os tipos de erros refracionais } \\
\hline Dioptria & Miopia & Hipermetropia & Astigmatismo miópico & Astigmatismo hipermetrópico \\
\hline$<1 \mathrm{D}$ & $12,3 \%$ & $11,1 \%$ & $23,4 \%$ & $23,2 \%$ \\
\hline 1 a $2,75 \mathrm{D}$ & $65,9 \%$ & $70,5 \%$ & $76,5 \%$ & $66,9 \%$ \\
\hline 3 a $4,75 \mathrm{D}$ & $78,4 \%$ & $72,6 \%$ & $71,0 \%$ & $52,8 \%$ \\
\hline 5 a $6,75 \mathrm{D}$ & $80,8 \%$ & $21,4 \%$ & $29,6 \%$ & $12,3 \%$ \\
\hline 7 a $9,75 \mathrm{D}$ & $70,8 \%$ & $3,4 \%$ & $7,7 \%$ & $2,0 \%$ \\
\hline 10 a $12,75 \mathrm{D}$ & $63,7 \%$ & $1,2 \%$ & $3,2 \%$ & $0,8 \%$ \\
\hline 13 a $15,75 \mathrm{D}$ & $6,0 \%$ & $0,6 \%$ & $1,2 \%$ & $0,8 \%$ \\
\hline $16 \mathrm{D}$ ou maior & $2,2 \%$ & $0,6 \%$ & $0,6 \%$ & $0,6 \%$ \\
\hline
\end{tabular}

LadarVision ${ }^{\circledR}$ e Meditec MEL-70 ${ }^{\circledR}$, ambos com 11,7\%, LaserSight LSX ${ }^{\circledast}(9,9 \%)$, VisX 20/20 ${ }^{\circledast}(0,7 \%)$, WaveLight Allegretto $^{\circledast}(0,2 \%)$, e outros modelos de excimer laser $(15,4 \%)$.

O microceratótomo mais utilizado é o Hansatome ${ }^{\circledR}(57,7 \%)$, seguido pelo Moria Carriazo-Barraquer ${ }^{\circledR}(19,2 \%)$, ACS $^{\circledR}$ $(18,5 \%)$, MK $2000^{\circledast}(17,1 \%)$, LSK One ${ }^{\circledast}(8,7 \%)$, Summit Krumeich-Barraquer ${ }^{\circledR}(7,8 \%)$, Amadeus ${ }^{\circledR}(5,3 \%)$, Moria One disposable $^{\circledast}(4,3 \%)$, LaserSight disposable ${ }^{\circledast}(1,7 \%)$, Inovatome ${ }^{\circledast}$ $(0,4 \%)$, e outros $(5,0 \%)$.

\section{Práticas padrão}

\section{Lamela}

Se não houver restrição, a plataforma de corte preferida dos respondentes é a de $160 \mu \mathrm{m}(75,5 \%)$, seguida pela de $180 \mu \mathrm{m}(14,2 \%)$ e a de $130 \mu \mathrm{m}(8,5 \%)$. Outros preferem as plataformas de 130 e $160 \mu \mathrm{m}(0,4 \%)$ e 160 e $180 \mu \mathrm{m}(1,3 \%)$. O diâmetro preferido da lamela corneana pediculada para a correção da miopia é de $8,5 \mathrm{~mm}(75,7 \%)$, seguido pelo de $9,5 \mathrm{~mm}$ (21,9\%). Outros diâmetros representaram 2,4\% das respostas. Para a correção da hipermetropia, o diâmetro da lamela corneana pediculada preferido foi de $9,5 \mathrm{~mm}(82,2 \%)$, seguido pelo de $8,5 \mathrm{~mm}(16,6 \%)$. Outros diâmetros representaram 1,2\%.

\section{Vestimenta}

Durante a cirurgia, 95,0\% dos entrevistados usam máscara cirúrgica, $16,8 \%$ usam luvas estéreis normais, $14,1 \%$ usam luvas estéreis de látex, $0,7 \%$ usam luvas estéreis com talco, $54,3 \%$ usam "sterile drap", 4,2\% usam "non sterile drap", 98,1\% usam gorro cirúrgico, $87,2 \%$ usam avental cirúrgico e 95,2\% usam protetores cirúrgicos estéreis para os pés.

\section{Leito estromal residual}

Durante o primeiro procedimento, leito estromal residual mínimo (LEM) maior ou igual a $300 \mu$ m é preferido por $30,6 \%$ dos respondentes; leito estromal mínimo entre 275 e $299 \mu \mathrm{m}$, por 11,8\%; LEM entre 250 e $274 \mu \mathrm{m}$, por 51,3\%; LEM entre 225 e $249 \mu \mathrm{m}$, por $4,5 \%$; LEM entre 200 e $224 \mu \mathrm{m}$, por $1,9 \%$; e nenhum dos respondentes utiliza LEM menor ou igual a $200 \mu \mathrm{m}$ no primeiro procedimento. Durante uma segunda intervenção, LEM maior ou igual a $300 \mu \mathrm{m}$ é aceita por $15,7 \%$ dos respondentes; LEM entre 275 e $299 \mu \mathrm{m}$, por 15,2\%; LEM entre 250 e $274 \mu \mathrm{m}$, por $54 \%$; LEM entre 225 e $249 \mu \mathrm{m}$, por $10,1 \%$; LEM entre 200 e $224 \mu \mathrm{m}$, por $3,8 \%$; e LEM menor ou igual a $200 \mu \mathrm{m}$, por $0,5 \%$ dos entrevistados.

\section{Ceratometria}

A menor ceratometria em que realizaria LASIK miópico seria maior ou igual a $38 \mathrm{D}$ para $42,4 \%$ dos entrevistados; entre 36 e 38 D para $33,3 \%$; entre 33 e 35 D para $19,2 \%$; entre 30 e $32 \mathrm{D}$ para $5,0 \%$; e menor ou igual a $30 \mathrm{D}$ para $0,2 \%$. Para a correção da hipermetropia com excimer laser, a maior ceratometria em que realizaria LASIK seria entre 45 e 47 D para $71,6 \%$ dos entrevistados; entre 48 e $50 \mathrm{D}$ para $26,7 \%$; e maior ou igual a $50 \mathrm{D}$, para $1,7 \%$ dos oftalmologistas.

\section{Diâmetro pupilar}

O maior diâmetro pupilar na penumbra em que realizariam LASIK: seria $8 \mathrm{~mm}$ para 9,2\% dos respondentes; $7 \mathrm{~mm}$ para $36,6 \% ; 6 \mathrm{~mm}$ para $42,1 \% ; 5 \mathrm{~mm}$ para $11,3 \%$; e maior que $8 \mathrm{~mm}$ para $0,8 \%$ dos entrevistados.

\section{LASIK vS PRK}

O gráfico 1 mostra a porcentagem que o LASIK ou PRK representam na prática cirúrgica refrativa dos respondentes.

\section{Cirurgia seqüiencial vs simultânea}

O gráfico 2 mostra a preferência dos entrevistados quanto à realização de cirurgias refrativas a laser de modo seqüencial (em dias diferentes) ou simultânea (no mesmo dia).

\section{Monovisão}

Quarenta por cento dos respondentes não realizam monovisão em suas cirurgias refrativas a laser, $47,5 \%$ realizam mo-

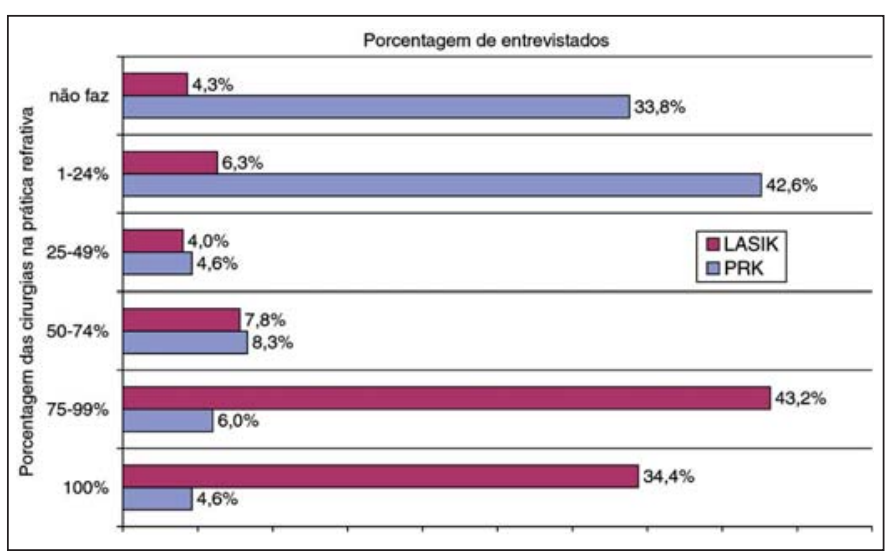

Gráfico 1 - Representação de quanto o LASIK ou PRK representam na prática refrativa 


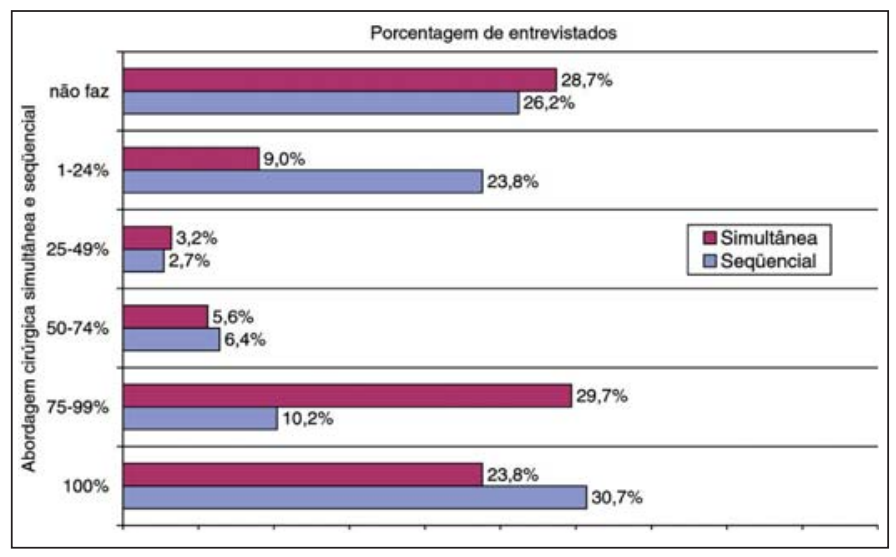

Gráfico 2 - Preferência dos entrevistados quanto à realização de cirurgias refrativas com excimer laser de modo seqüencial ou simultâneo

novisão em 1,0 a 24,0\% dos pacientes; $4,5 \%$ realizam em 25,0 a $49,0 \% ; 3,7 \%$ realizam em 50,0 a 74,0\%; 3,7\% realizam em 75,0 a $99,0 \%$; e $1,7 \%$ realizam em $100 \%$ dos pacientes. A melhor correção para a monovisão seria de $1,5 \mathrm{D}$ para $24,8 \%$ dos respondentes; menor que $1,5 \mathrm{D}$ para $14,8 \%$; $2 \mathrm{D}$ para $12,5 \%$; e $1,75 \mathrm{D}$ para $10,5 \%$ dos entrevistados.

\section{Lâminas}

Seis por cento dos entrevistados relataram que trocam a lâmina do microceratótomo entre diferentes olhos; $32,5 \%$ referem trocar entre pacientes; $40,7 \%$ trocam-na entre 2 a 3 pacientes; $15,8 \%$ trocam-na entre 4 a 6 pacientes; $2,9 \%$ trocam-na entre 7 a 10 pacientes; $0,9 \%$ trocam-na entre mais de 10 pacientes; e 1,2\% trocam-na diariamente.

\section{Medicação pós-operatória}

O quadro 3 mostra a distribuição dos tipos de medicamen- tos utilizados no pós-operatório das cirurgias refrativas a laser, de acordo com os entrevistados. O quadro 4 mostra a porcentagem dos entrevistados em relação ao tempo de uso da medicação pós-operatória em LASIK.

\section{Avaliação pós-operatória}

O exame pós-operatório é realizado imediatamente após a cirurgia por $19,2 \%$ dos entrevistados; $11,1 \%$ examinam o paciente antes de o mesmo ir embora; $37,9 \%$ avaliam o paciente no primeiro dia após a cirurgia; $1,2 \%$ examinam os pacientes imediatamente após a cirurgia e antes deles irem embora; $15,9 \%$ examinam imediatamente após a cirurgia e no dia seguinte; $8,7 \%$ examinam antes de os pacientes irem embora e no dia seguinte; e 5,2\% examinam imediatamente após a cirurgia, antes de os pacientes irem embora e no dia seguinte. Em 0,9\% dos respondentes, o pós-operatório é realizado por assistente.

\section{Complicações e efeitos colaterais}

A tabela 1 mostra a distribuição das complicações e efeitos colaterais de acordo com os respondentes.

\section{Wavefront}

"Wavefront" é utilizado por 4,5\% dos entrevistados, 95,5\% referem não usá-lo, porém 76,4\% acreditam que o "wavefront" proporcionará melhora da qualidade de visão.

\section{Custo}

O gráfico 3 mostra o custo da cirurgia refrativa a laser, de acordo com os respondentes. O gráfico 4 mostra a perspectiva dos respondentes em relação à mudança no preço do LASIK. A tabela 2 mostra a distribuição no modo de pagamento das cirurgias.

Dos respondentes, 68,4\% não são sócios da SBCR.

Quadro 3. Distribuição dos tipos de medicações utilizadas no per-operatório de cirurgias refrativas a laser, segundo os respondentes

\begin{tabular}{|c|c|c|c|c|c|c|}
\hline \multirow[t]{2}{*}{ Medicamento } & \multicolumn{2}{|c|}{ Pré-operatório } & \multicolumn{2}{|c|}{ Intra-operatório } & \multicolumn{2}{|c|}{ Pós-operatório } \\
\hline & Não usa & Usa & Não usa & Usa & Não usa & Usa \\
\hline Fluorquinolona & & $19,9 \%$ & & $29,9 \%$ & & $46,4 \%$ \\
\hline Sulfonamida & & $0,9 \%$ & & $0,3 \%$ & & $1,2 \%$ \\
\hline Fluorquinolona+aminoglicosídeo & & $1,9 \%$ & & $4,2 \%$ & & $6,9 \%$ \\
\hline Fluorquinolona+sulfonamida & & $0,2 \%$ & & $0,2 \%$ & & $0,2 \%$ \\
\hline Esteróides & $86,5 \%$ & $13,5 \%$ & $63,0 \%$ & $37,0 \%$ & $8,9 \%$ & $91,1 \%$ \\
\hline Médios & & $10,5 \%$ & & $26,4 \%$ & & $67,9 \%$ \\
\hline Fortes & & $3,0 \%$ & & $10,6 \%$ & & $23,2 \%$ \\
\hline Não-hormonais & $86,5 \%$ & $13,5 \%$ & $76,9 \%$ & $23,1 \%$ & $62,8 \%$ & $37,2 \%$ \\
\hline Com preservativo & & $7,0 \%$ & & $12,1 \%$ & & $18,8 \%$ \\
\hline Sem preservativo & & $6,5 \%$ & & $11,0 \%$ & & $18,4 \%$ \\
\hline
\end{tabular}




\begin{tabular}{|c|c|c|c|c|}
\hline \multicolumn{5}{|c|}{$\begin{array}{l}\text { Quadro 4. Porcentagem dos entrevistados em relação ao tempo de } \\
\text { uso de medicação pós-operatória em LASIK }\end{array}$} \\
\hline $\begin{array}{l}\text { Tempo } \\
\text { de uso }\end{array}$ & Antibiótico & NSAID & $\begin{array}{l}\text { Lágrima } \\
\text { artificial }\end{array}$ & Esteróide \\
\hline$<1$ dia & $0,5 \%$ & $12,4 \%$ & - & $0,4 \%$ \\
\hline 2-7 dias & $61,5 \%$ & $50,9 \%$ & $5,2 \%$ & $39,6 \%$ \\
\hline 8-30 dias & $37,2 \%$ & $32,4 \%$ & $34,3 \%$ & $49,7 \%$ \\
\hline $1-3$ meses & $0,3 \%$ & $3,8 \%$ & $39,3 \%$ & $9,9 \%$ \\
\hline$>3$ meses & $0,2 \%$ & $0,5 \%$ & $21,2 \%$ & $0,4 \%$ \\
\hline \multicolumn{5}{|c|}{ NSAID= "non steroid antinflammatory drug" } \\
\hline
\end{tabular}

\section{DISCUSSÃO}

\section{Fatores demográficos}

Esta pesquisa teve uma taxa de respondentes similar às últimas realizadas pela ASCRS, dentro dos Estados Unidos: 985 respondentes $(15 \%)^{(10)}$ e 509 respondentes $(11 \%)^{(12)}$, e com sócios membros de todo o mundo: 1.174 respondentes $(13,2 \%)^{(9)}$. No Brasil, 7,2\% dos respondentes desta pesquisa já se submeteram a algum tipo de CR, comparado a outro estudo $^{(13)}$, em que $17,6 \%$ dos respondentes já se submeteram a alguma CR. Em alguns estudos recentes, a maioria dos respondentes tinha entre 40 e $54 \operatorname{anos}^{(13)}$ e entre 40 e 59 anos $^{(10)}$. Em $2004^{(13)}$, o grupo com maior experiência em CR, mais de $25 \%$ dos cirurgiões refrativos respondentes, tinha entre 7 e 10 anos de experiência refrativa.

\section{Número de cirurgia refrativa, tipos de cirurgia refrativa e local onde as realizam}

Estes autores ${ }^{(13)}$ também referem que mais da metade $(53 \%$, contra $51,2 \%$ da pesquisa anterior ${ }^{(9)}$ ) dos respondentes realizaram até 20 cirurgias refrativas por mês; 19,6\% (contra $19,7 \%$ da pesquisa anterior ${ }^{(9)}$ ) realizaram menos que 5 ; e que $47 \%$ (contra $48,8 \%$ da pesquisa anterior ${ }^{(9)}$ ) realizaram mais de 20 CRs por mês. É referido ${ }^{(13)}$ também que há mais cirurgiões dentre os que já se submeteram a alguma CR (84\%) do que os que nunca se submeteram $(53,6 \%)$. O contrário, também reforça esta correlação: enquanto $15,2 \%$ dos que já se submeteram a algum tipo de CR não realizaram CR; 46,4\% dos que nunca se submeteram à CR não a realizaram ${ }^{(13)}$. Assim como

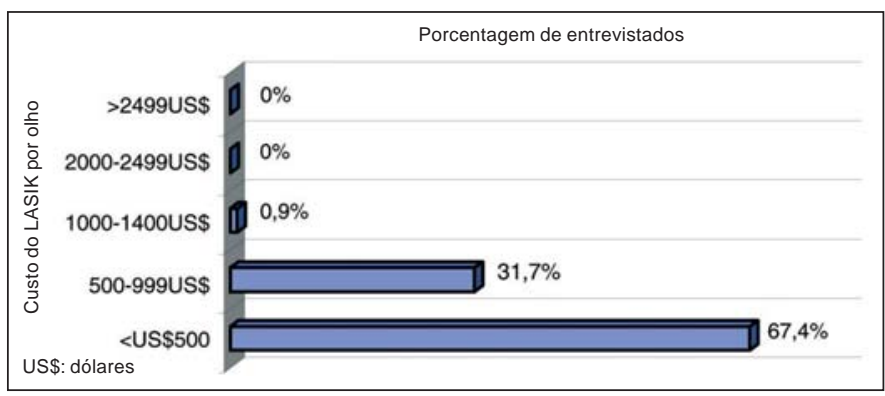

Gráfico 3 - Custo do LASIK por olho (em dólares) segundo os respondentes

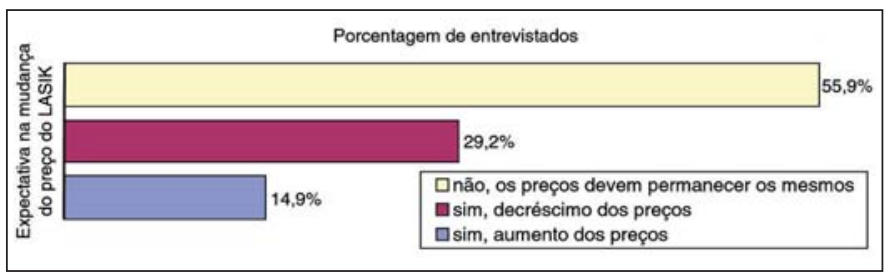

Gráfico 4 - Expectativa de mudança no preço do LASIK nos 6 meses seguintes

no Brasil, o LASIK é o procedimento refrativo preferido dos cirurgiões $^{(9-10,13)}$. Pesquisas de $2003^{(10)}$ referem que a porcentagem de respondente que fazem LASIK estaria estável, em torno de 55 a $56 \%$, desde $2001^{(14-15)}$. O LASEK foi realizado por $26 \%$ dos respondentes em $2003^{(10)}$, contra $13 \%$ em $2002^{(15)}$. Em $2003^{(10)}$, o PRK foi realizado por $47 \%$, anel intra-corneano por $4 \%$, ceratoplastia lamelar térmica por $2 \%$, ceratoplastia condutiva por $10 \%$, implantes fácicos por $7 \%$, extração de cristalino transparente por $40 \%$, incisões relaxantes por $78 \%$ dos entrevistados. Estes autores ${ }^{(10)}$ referem ainda que dos que utilizaram o excimer laser, $16 \%$ utilizaram o de um centro refrativo aberto para "locação", $40 \%$ têm seus próprios excimers, e $44 \%$ utilizaram os excimers dos quais são sócios.

\section{LASIK}

Quanto aos exames pré-operatórios, a topografia corneana também é realizada pela grande maioria dos respondentes de uma pesquisa similar ${ }^{(13)}$. Que também revela que $89,3 \%$ de seus respondentes realizam a medida direta do diâmetro pupilar no escuro, e que 44,1\% utilizam o pupilômetro infraverme-

\begin{tabular}{|c|c|c|c|c|c|c|}
\hline $\begin{array}{l}\text { Freqüência de } \\
\text { aparecimento }\end{array}$ & Olho seco & $\begin{array}{c}\text { Ceratite difusa } \\
\text { da interface }\end{array}$ & $\begin{array}{l}\text { Epitelização } \\
\text { da interface }\end{array}$ & "Glare" & Infecção & Estrias no "flap" \\
\hline Não tem & $11,8 \%$ & $28,6 \%$ & $39,5 \%$ & $13,5 \%$ & $82,4 \%$ & $17,0 \%$ \\
\hline $1-4 \%$ & $17,9 \%$ & $23,6 \%$ & $15,9 \%$ & $28,4 \%$ & $1,4 \%$ & $29,6 \%$ \\
\hline $5-14 \%$ & $20,7 \%$ & $8,6 \%$ & $3,3 \%$ & $22,3 \%$ & $0,6 \%$ & $14,5 \%$ \\
\hline $15-24 \%$ & $17,3 \%$ & $2,9 \%$ & $1,0 \%$ & $12,9 \%$ & $0,2 \%$ & $6,2 \%$ \\
\hline $50 \%$ & $10,3 \%$ & $0,6 \%$ & $0,4 \%$ & $3,3 \%$ & - & $0,9 \%$ \\
\hline
\end{tabular}




\begin{tabular}{|lrr|}
\hline \multicolumn{3}{|l|}{ Tabela 2. Distribuição do modo de pagamento das cirurgias refra- } \\
tivas a laser \\
\hline $\begin{array}{l}\text { Freqüência do } \\
\text { modo de pagamento }\end{array}$ & Particular & Convênio \\
$1-10 \%$ & $11,0 \%$ & $15,21 \%$ \\
$11-20 \%$ & $8,4 \%$ & $12,39 \%$ \\
$21-30 \%$ & $10,7 \%$ & $7,39 \%$ \\
$31-40 \%$ & $4,4 \%$ & $4,56 \%$ \\
$41-50 \%$ & $8,9 \%$ & $9,78 \%$ \\
$51-60 \%$ & $5,9 \%$ & $8,69 \%$ \\
$61-70 \%$ & $5,7 \%$ & $8,69 \%$ \\
$71-80 \%$ & $7,8 \%$ & $13,69 \%$ \\
$81-90 \%$ & $13,5 \%$ & $14,34 \%$ \\
$91-100 \%$ & $23,6 \%$ & $5,21 \%$ \\
\hline
\end{tabular}

lho. O teste de Schirmer é realizado por 34,5\% dos respondentes ${ }^{(13)}$, enquanto neste estudo, por $14,1 \%$.

Ao contrário desta pesquisa, outros autores revelam que o excimer laser mais utilizado é o Visx ${ }^{\circledR}$, variando de $57,6 \%{ }^{(13)}$ a $71 \%^{(10)}$ ou $72 \%{ }^{(12)}$; seguido pelo LadarVision ${ }^{\circledR}$ com $17 \%{ }^{(10,12-13)}$. Em seguida, empatados, estão os excimers Technolas Bausch \& Lomb $^{\circledR}$ e Nidek $^{\circledR}$ com $4 \%{ }^{(10,12)}$. Um estudo recente ${ }^{(13)}$ revela que o excimer laser preferido dos latinos americanos é o $\mathrm{Nidek}^{\circledR} \mathrm{NC}$ 5000 , fato que coincide em parte com esta pesquisa, pois foi avaliado apenas o Brasil. Assim como no Brasil, o microcerátomo (MK) mais utilizado é o Hansatome ${ }^{\circledR(10,12-13)}$. Em segunda e terceira posição de preferência, estão Amadeus ${ }^{\circledR} \mathrm{e}$ Moria Carriazo-Barraquer $^{\circledR(10,13)}$. Segundo este último estudo ${ }^{(13)}$ os MKs que obtiveram maior aumento de preferência foram o IntraLaser ${ }^{\circledR}$ (de $2,3 \%$ para $5,1 \%)$ e o $\mathrm{BD}^{\circledR}$ (de $1,8 \%$ a $\left.3,1 \%\right)$. Em relação ao diâmetro do disco, este estudo ${ }^{(13)}$ revela que para os míopes a preferência é a mesma para os discos de 8,5 e 9,5 $\mathrm{mm}$ de diâmetro (35\%), enquanto para os hipermétropes, o diâmetro preferido é semelhante ao presente estudo, $82,4 \%$ preferem diâmetro de $9,5 \mathrm{~mm}$, contra $14 \%$ que preferem o de $8,5 \mathrm{~mm}$.

Enquanto 100\% dos respondentes africanos usam máscara cirúrgica durante o LASIK, os da Ásia, Canadá, Europa, América Latina, Oceania e Estados Unidos (EUA), usam máscara cirúrgica com menor freqüência que os brasileiros, $89,3 \%$, $71,4 \%, 66,7 \%, 85,7 \%, 69,2 \%$, e $77,9 \%$, respectivamente ${ }^{(13)}$. Entretanto, mesmo somando os resultados dos respondentes que referem usar algum tipo de luva no presente estudo $(31,6 \%)$, a freqüência de uso de luvas na cirurgia do LASIK no Brasil (em 2002) só ganha para a dos respondentes africanos, que não as usam ${ }^{(13)}$, pois na Ásia, Canadá, Europa, Oceania e EUA a freqüência de uso de algum tipo de luvas cirúrgica é de $64,3 \%, 57,1 \%, 71,6 \%, 92,3 \%$, e 64,3\%(13). É apenas comparável ao dos latinos americanos, que é de $28,5 \%^{(13)}$. Quanto ao uso de gorro cirúrgico nas cirurgias de LASIK, que no Brasil é quase uma unanimidade com 98,1\%, este achado só é similar aos dos africanos, onde $100 \%$ os usam durante o LASIK ${ }^{(13)}$. Pois os respondentes de Ásia, Canadá, Europa, América Latina, Oceania e EUA referiram usar gorro cirúrgica com menor freqüência: $82,1 \%, 50 \%, 46,7 \%, 81 \%, 76,9 \%$, e $80,2 \%$, respecti- vamente $^{(13)}$. Durante o LASIK, os cirurgiões latino americanos são os que usam com maior frequiência avental cirúrgico $(71,4 \%)^{(13)}$, dado mais próximo da freqüência no Brasil, que é de $87,2 \%$. A frequiência de uso desta vestimenta na África, Ásia, Canadá, Europa, Oceania e Estados Unidos é de 50\%, $53,6 \%, 0 \%, 43,3 \%, 46,2 \%$, e 6,7\%, respectivamente ${ }^{(13)}$. Em $2001^{(16)}$, os sul-americanos usaram com menor freqüência luvas durante o LASIK do que a Europa, Oceania e EUA; porém usam mais freqüentemente avental cirúrgico que estas outras localidades. Em outra pesquisa mais atual ${ }^{(13)}$, os latinos americanos são alguns dos que usam máscara cirúrgica, gorro cirúrgico e avental cirúrgico com maior freqüência do que a maior parte do mundo, porém são alguns dos que menos usam luvas cirúrgicas para o LASIK.

Assim como no presente estudo, a maioria dos respondentes de outro mais recente ${ }^{(13)} \mathrm{e}$ de mesma época ${ }^{(16)}$ preferem LEM entre 250 e $274 \mu \mathrm{m}$, durante o primeiro ou segundo tratamentos, porém em maior porcentagem. Apesar de haver certo consenso de LEM em cirurgias primárias de LASIK ${ }^{(8)}, 6,4 \%$ dos entrevistados brasileiros ainda deixam LEM menor que este, dado semelhante à pesquisa internacional similar da mesma época ${ }^{(16)}$, onde $5 \%$ dos entrevistados iam além dos $250 \mu \mathrm{m}$. Entretanto, esta freqüência baixou para $1,1 \%$ mais recentemente ${ }^{(13)}$.

Em relação à ceratometria, uma pesquisa ${ }^{(16)}$ similar, da mesma época deste, refere que $45 \%$ dos respondentes programam, para LASIK miópico, ceratometria central corneana mais plana entre 33 e 35 D, e, para LASIK hipermetrópico, ceratometria entre 45 e 47 D. Mais recentemente ${ }^{(13)}$, estes valores mudaram para: 36 e 38 D; e 48 e $50 \mathrm{D}$, respectivamente. Em contraste com o presente estudo, outro ${ }^{(16)}$ revela que $99 \%$ dos entrevistados realizariam LASIK em pacientes com diâmetro pupilar na penumbra de $6 \mathrm{~mm}$, e $34 \%$ fariam em pacientes com diâmetro pupilar na penumbra de $8 \mathrm{~mm}$.

Ao contrário dos achados deste estudo, outro autor ${ }^{(10)}$ refere que apenas $2 \%$ dos respondentes não realizam LASIK de modo simultâneo (os dois olhos no mesmo dia), e que em 1998 este percentual foi de 17\%. Enquanto 99\% destes mesmos respondentes referiram sempre realizar LASIK de modo simultâneo em 2003, este percentual foi de $21 \%$ em 1998, 52\% em 2000, e $65 \%$ em 2001 ${ }^{(10)}$. Atualmente ${ }^{(13)}$, a prática do LASIK simultâneo ainda é a mais utilizada. Em relação à monovisão, um relato semelhante ${ }^{(13)}$ refere que $8,9 \%$ dos respondentes não realizam monovisão, e que $64,9 \%$ referem fazer monovisão em 1 a $24 \%$ dos seus pacientes. Estudos de 2002 a $2005^{(9,13,16)}$ referem que em torno de três quartos dos cirurgiões preferem trocar a lâmina do MK entre pacientes, entretanto cerca de um terço dos respondentes da Oceania e dois terços dos Europeus preferem trocar a lâmina do MK entre olhos. Nestes $\operatorname{estudos}^{(9,13,16)}$, cerca de metade dos respondentes latinos americanos preferem trocar a lâmina do MK entre 2 ou mais pacientes. Assim como o presente estudo, em 2001 ${ }^{(16)}$ os antibióticos mais utilizados foram do grupo das fluorquinolonas, de maneira que $57 \%$ destes respondentes os utilizaram no pré-operató- 
rio, $78 \%$ no per-operatório, e $84 \%$ no pós-operatório. Porém, mais recentemente ${ }^{(12-13)}$, as fluorquinolonas de quarta geração foram as que apresentaram maior aumento relativo de preferência. Em sincronia com o presente estudo, em $2001^{(16)}$, no pós-operatório, os esteróides de média potência foram os mais utilizados. Todavia, estudo mais recente ${ }^{(10)}$ refere que, no pósoperatório, a prednisolona é o agente esteróide mais utilizado. Entre as complicações, o olho seco é a mais frequiente desde 2001, seguido pelo "glare"(9,13,16). O uso da tecnologia de "wavefront" pelos brasileiros está similar à referida por um estudo semelhante da mesma época ${ }^{(16)}$. Este ${ }^{(16)}$ refere que o "wavefront" foi utilizado por até 3\% dos respondentes de todos os países, excetuando os europeus, onde a freqüência de uso foi de $14 \%$. Apesar de cerca de $98 \%$ dos respondentes disseram em 2001 que não utilizam ablação customizada, 77\% deles referiram que acreditam que esta tecnologia pode aumentar a qualidade de visão ${ }^{(16)}$. O custo do LASIK vem se mantendo entre US\$ 1.500 e US\$ 2.000 por olho nos EUA, Europa e Oceania, entre 2002 e $2005^{(9,13,16)}$. Na América Latina, o custo por olho do LASIK vem se mantendo entre os mais baixos do mundo ${ }^{(9,13,16)}$, sendo que $25 \%$ dos latinos americanos referem, atualmente ${ }^{(13)}$, praticar preços menores que US\$ 500 por olho.

Assim como as maiores e mais divulgadas pesquisas deste tipo no mundo ${ }^{(9-16)}$, esta tem o objetivo principal de tornar mais acessível este tipo informação para todos os oftalmologistas. Desta forma, pode-se observar e monitorar aspectos demográficos, práticas padrões, tendências, tecnologia e mercado da CR.

\section{ABSTRACT}

Purpose: To perform the first Brazilian refractive surgery survey. Methods: Between August 2001 and February 2002, a questionnaire was mailed to 7890 Brazilian ophthalmologists. The questionnaire presented questions about demographic aspects, technology, instruments, market, trends, practice patterns and cost of refractive surgery. Results: Nine hundred and twenty questionnaires (11.67\%) were answered. LASIK is the preferred technique for correction between +5.00 and -7.00 D. The mostly used excimer laser is Nidek ${ }^{\circledR}$ EC 5000, and Hansatome is the mostly used microkeratome. Others aspects like: demographic, techniques, practice patterns, trends, market, and pre-, intra- and postoperative care, were analyzed. Conclusion: With this survey, the Brazilian ophthalmologists can observe and monitor these aspects of refractive surgery in Brazil, and compare them with others surveys.

Keywords: Refractive errors/surgery; Laser surgery; Keratomileusis, laser in situ; Questionnaires; Ophthalmology/statistics \& numerical data; Physician's practice patterns/statistics $\&$ numerical data; Data collection; Brazil

\section{REFERÊNCIAS}

1. Barraquer JI. Queratoplastia refractiva. Estud Inform Oftalmol Inst Barraquer. 1949;10(1):2-21.

2. Barraquer JI. Keratomileusis. Int Surg. 1967;48(2):103-17.

3. Barraquer JI. Lamellar keratoplasty. (Special techniques). Ann Ophthalmol. 1972;4(6):437-69.

4. Barraquer JI. Method for cutting lamellar grafts in frozen corneas: New orientations for refractive surgery. Arch Soc Am Ophthalmol. 1958;1:237.

5. Barraquer JI. Results of myopic keratomileusis. J Refract Surg. 1987;3(1):98-101.

6. Barraquer JI. Queratomileusis para la correction de la miopia. Arch Soc Am Oftal Optom. 1964;5(1):27-48

7. Pallikaris IG, Papatzanaki ME, Stathi EZ, Frenschock O, Georgiadis A. Laser in situ keratomileusis. Lasers Surg Med. 1990;10(5):463-8.

8. Sugar A, Rapuano CJ, Culbertson WW, Huang D, Varley GA, Agapitos PJ, et al. Laser in situ keratomileusis for myopia and astigmatism: safety and efficacy: a report by the American Academy of Ophthalmology. Ophthalmology. 2002;109(1):175-87.

9. Solomon KD, Fernandez de Castro LE, Sandoval HP, Bartholomew LR, Vroman DT. Refractive Surgery Survey 2003. J Cataract Refract Surg. 2004; 30(7): 1556-69.

10. Leaming DV. Practice styles and preferences of ASCRS members-2003 Survey. J Cataract Refractive Surg. 2004;30(4):892-900.

11. Leaming DV. Practice styles and preferences of ASCRS members-1985 Survey. J Cataract Refract Surg. 1986;12(4):380-4.

12. Duffey RJ, Leaming D. Trends in refractive surgery in the United States. J Cataract Refract Surg. 2004;30(8):1781-5.

13. Sandoval HP, de Castro LE, Vroman DT, Solomon KD. Refractive Surgery Survey 2004. J Cataract Refract Surg 2005;31(1):221-33.

14. Leaming DV. Practice styles and preferences of ASCRS members-2001 Survey. J Cataract Refract Surg. 2002;28(9):1681-8.

15. Leaming DV. Practice styles and preferences of ASCRS members-2002 Survey. J Cataract Refract Surg. 2003;29(7):1412-20.

16. Solomon KD, Holzer MP, Sandoval HP, Vargas LG, Werner L, Vroman DT, et al. Refractive Surgery Survey 2001. J Cataract Refract Surg. 2002; 28(2):346-55. 